\title{
SENTIDO DE AGENCIA Y SUS DISRUPCIONES EN LA DEPRESIÓN: UNA PERSPECTIVA INTERDISCIPLINAR
}

\author{
SENSE OF AGENCY AND ITS DISRUPTIONS IN DEPRESSION: AN \\ INTERDISCIPLINARY APPROACH
}

\author{
Penélope Pereira-Perdomo* \\ Universidad de Chile \\ Santiago-Chile \\ Bryan Zúñiga-Iturra \\ Universidad de Chile \\ Santiago-Chile \\ Recibido abril de 2020/Received April, 2020 \\ Aceptado agosto de 2020/Accepted August, 2020
}

\begin{abstract}
RESUMEN
El presente artículo tiene como objetivo principal analizar las alteraciones en el sentido de agencia en personas con trastorno depresivo mayor o depresión clínica por medio del estudio de testimonios de pacientes registrados en la literatura. Nuestro plan de trabajo seguirá el siguiente esquema. Tras la sección introductoria, en el primer apartado describiremos la aproximación a la depresión por parte del enfoque nosológico, representado por manuales diagnósticos como el DSM. Sostendremos que este es insuficiente por, al menos, tres motivos: (i) reduce la psicopatología a una sintomatología, (ii) se enfoca en síntomas que poseen una naturaleza heterogénea, por lo que se integran de manera confusa al cuadro total del desorden, y (iii) minimiza la subjetividad. Tomando en cuenta estas limitaciones, presentaremos las características del método propio de la psiquiatría fenomenológica como una alternativa al enfoque exclusivamente nosológico. En el segundo apartado caracterizaremos los desórdenes de la corporización y, en particular, el sentido de agencia. En el tercer apartado identificaremos cuatro rasgos que dan cuenta de la disrupción de dicho sentido en la depresión, a saber: (i) pérdida de control, (ii) pasividad patológica, (iii) chrematización e (iv) inhibición. Para finalizar, puntualizaremos las implicancias conceptuales y metodológicas de este análisis en el estudio integral de los trastornos mentales.

Palabras Clave: Sentido de agencia, Depresión, Nosología, Corporización, Fenomenología.
\end{abstract}

\begin{abstract}
The following article aims to analyze the alterations in the sense of agency in people with major depressive disorder or clinical depression through the study of patient's testimonies registered in the literature. We will pursue the following scheme. After the introductory section, the first section describes the approximation of the nosological approach of depression represented by diagnostic manuals such as DSM. We will claim that this approach is insufficient for, at least, three reasons: (i) it reduces psychopathology to symptomatology, (ii) it focuses on heterogeneous symptoms, which causes a confusing integration in the total picture of the disorder, and (iii) it minimizes subjectivity. Considering these limitations, we will present the characteristics of this method of phenomenological psychiatry as an alternative to the exclusively nosological approach. In the second section, we will describe the disturbances of embodiment and, in particular, the sense of agency. In the third section, we will identify four aspects that show disruption in that sense in depression, namely: (i) loss of control, (ii) pathological passivity, (iii) chrematization, and (iv) inhibition. Finally, we will point out the conceptual and methodological implications of this analysis in the comprehensive study of mental disorders.
\end{abstract}

Key Words: Sense of Agency, Depression, Nosology, Embodiment, Phenomenology. 


\section{INTRODUCCIÓN}

El sentido de agencia es definido como la experiencia de control en el inicio o generación de una acción propia, es decir, el reconocimiento de uno mismo como su agente. Corresponde a un fenómeno cognitivo complejo que forma parte de la autoconsciencia básica -una consciencia de sí prerreflexiva y de primer orden-. Como tal, su abordaje ha sido llevado a cabo desde la interdisciplinariedad. Uno de sus principales focos de desarrollo teórico en la actualidad se encuentra ligado a la noción de corporización, esto es, la consideración del cuerpo (incluido el cerebro) y el ambiente como elementos constituyentes de los procesos cognitivos. En su estudio convergen, entre otras, la fenomenología y el enfoque de la cognición corporizada (embodied cognition) en ciencias cognitivas, los que conforman una perspectiva que ha adquirido centralidad en psiquiatría.

La importancia del sentido de agencia es notoria en la vida cotidiana, pues se vincula con el repertorio conductual, la responsabilidad y el desenvolvimiento en el entorno. Preliminarmente, parece constituir un aspecto relevante a considerar en la práctica clínica, debido a que la noción de desorden mental apunta a una alteración del estado cognitivo y el comportamiento en las esferas personales, sociales, o laborales. No obstante, manuales diagnósticos como el Diagnostic and Statistical Manual of Mental Disorders (DSM) no refieren a dicho rasgo. Su enfoque nosológico y operacionalista se limita a ofrecer una sintomatología -es decir, un resumen de los síntomas característicos de los trastornos listados- que omite la autoconsciencia básica. Tal es el caso del trastorno depresivo mayor o depresión clínica, en donde las alteraciones en el sentido de agencia son una parte primordial no tomada en cuenta.

Con base en lo anterior, el objetivo del presente artículo radica en la realización de un análisis de las alteraciones en el sentido de agencia en sujetos con depresión por medio del estudio de testimonios de pacientes registrados en la literatura. La hipótesis de trabajo adoptada sostiene que estas alteraciones son centrales en la vivencia del desorden, y se expresan cabalmente por medio del material testimonial. Este material es fenomenológicamente relevante debido a su perspectiva en primera persona, que facilita el acceso a lo que la psiquiatría fenomenológica denomina el "modo de ser" del individuo afectado.
La ejecución del objetivo anteriormente planteado permite relacionar la importancia de la autoconsciencia y sus disrupciones en el trastorno mental con los alcances y límites del enfoque nosológico. La relevancia de la discusión puede ser localizada en dos ejes. En primer lugar, la centralidad de las distinciones fenomenológicas en una descripción de desórdenes como la depresión muestra las virtudes de la interdisciplinariedad en sus investigaciones. Los manuales de diagnóstico otorgan una visión estrecha, por lo que fallan en plasmar cabalmente los reportes y testimonios de los sujetos acerca de sus experiencias. Frente a ello, la aproximación interdisciplinar es un recurso provechoso, ya que posibilita un estudio de la enfermedad mental que aborda las estructuras y modos de ser subyacentes sin la reducción a la manifestación de un conjunto de síntomas.

En segundo lugar, la elección del trastorno depresivo mayor como desorden a analizar se justifica frente a las cifras nacionales de su prevalencia en la población. Respecto de este punto, según un informe del Centro de Estudios del Conflicto y la Cohesión Social (COES) y el Instituto Milenio para la Investigación en Depresión y Personalidad (MIDAP), uno de cada cinco chilenos presenta síntomas de depresión (COES 2018). De igual modo, de acuerdo con cifras de la Organización para la Cooperación y el Desarrollo Económicos (OCDE 2014), cerca del $90 \%$ de los suicidios en Chile están asociados a algún desorden mental -principalmente, trastornos depresivos-. La alta presencia de depresión y suicidio en nuestro país ha impulsado iniciativas como la Alianza Chilena Contra la Depresión, que trabaja desde el 2014 en el tratamiento del trastorno y la disminución de los actos suicidas e intentos de suicidio. Sin embargo, el índice de prevalencia continúa siendo elevado. $\mathrm{Si}$ a ello se suma la escasez de centros -de estudio o de terapia- que aborden la enfermedad desde una óptica fenomenológica, un análisis de este orden adquiere relevancia filosófica. En efecto, constituye una herramienta metodológica que nutre de nuevas alternativas tanto a los profesionales de la salud (psicólogos y psiquiatras) como a los pacientes.

El artículo seguirá el siguiente esquema. En el primer apartado abordaremos la aproximación a la depresión por parte del enfoque nosológico. Sostendremos que es insuficiente, en cuanto (i) reduce la psicopatología a una sintomatología, (ii) se centra en síntomas que son heterogéneos, por lo que se 
integran confusamente al cuadro total del desorden, y (iii) minimiza la subjetividad. A causa de sus limitaciones, presentaremos las características del método empleado por la psiquiatría fenomenológica para el análisis de la enfermedad mental como una alternativa. En el segundo apartado caracterizaremos los desórdenes de la corporización y, en particular, el sentido de agencia. En el tercer apartado determinaremos de qué manera dicho sentido se altera en el trastorno depresivo. Identificaremos cuatro rasgos que dan cuenta de su disrupción, a saber: (i) pérdida de control, (ii) pasividad patológica, (iii) chrematización e (iv) inhibición. Para finalizar, puntualizaremos las implicancias conceptuales y metodológicas del estudio de la corporización y sus alteraciones en el abordaje integral de los desórdenes mentales.

\section{UNA ALTERNATIVA AL ESTUDIO NOSOLÓGICO DEL TRASTORNO MENTAL: EL ANÁLISIS FENOMENOLÓGICO}

El estudio de las enfermedades mentales en la actualidad es llevado a cabo preponderantemente por la psiquiatría. Dicha aproximación opera desde un punto de vista nosológico y operacionalista, esto es, por medio de la descripción, categorización y diagnóstico de desórdenes por referencia a un conjunto de síntomas observables. Los desórdenes son descritos mediante características convencionales que ayuden a su clasificación (nosografía). En otros términos, hablar acerca de la enfermedad desde una perspectiva psiquiátrica supone establecer que un conjunto de manifestaciones (síntomas) se posicionan como condiciones de posibilidad de su padecimiento.

Los sistemas de clasificación empleados -principalmente, el $D S M-V$ - representan en gran medida qué significa el trastorno mental para la disciplina. De acuerdo con él (American Pyschiatric Association, 2013), corresponde a:

Un síndrome caracterizado por una alteración clínicamente significativa en la cognición, la regulación emocional o el comportamiento de un individuo, que refleja una disfunción en los procesos psicológicos, biológicos o del desarrollo que subyacen en su función mental. Los trastornos mentales suelen estar asociados a una angustia o discapacidad significativa, ya sea social, laboral o de otras actividades importantes (p. 20) $)^{1}$.
El $D S M-V$ aplica un modelo diagnóstico categorial. Según este, un trastorno es confirmado o descartado debido a la presencia de cierto número de signos (manifestaciones objetivas y observables) o síntomas (vivencias subjetivas concomitantes). Una ejemplificación de estas características es observada en su presentación del trastorno depresivo mayor, cuyo primer criterio enumera los siguientes síntomas:

1. Estado de ánimo deprimido.

2. Disminución del interés o placer por todas o la mayor parte de las actividades.

3. Pérdida o aumento de peso, disminución o aumento del apetito.

4. Insomnio o hipersomnia.

5. Agitación o retraso psicomotor.

6. Fatiga o pérdida de energía.

7. Sentimiento de inutilidad o culpabilidad excesiva o inapropiada.

8. Disminución de la capacidad de pensar o concentrarse, o indecisión.

9. Pensamientos de muerte recurrente, ideas suicidas recurrentes sin un plan determinado, intento de suicidio o un plan específico para llevarlo a cabo (op. cit., pp. 160-161).

El diagnóstico requiere que se presenten cinco o más de los síntomas mencionados casi todos los días en un período de dos semanas, y que influyan de manera negativa en el funcionamiento del sujeto. Al menos uno debe corresponder a (1) o (2).

Sin embargo, esta aproximación a la depresión es problemática por, al menos, tres razones ${ }^{2}$, que pueden aplicarse al DSM de manera general. En primer lugar, reduce la psicopatología a una sintomatología. En efecto, decanta en la presentación de un resumen de los síntomas, que son seleccionados por su relevancia en la identificación de categorías diagnósticas. Ello constituye una simplificación de los perfiles del trastorno. En segundo lugar, los síntomas elegidos presentan una naturaleza heterogénea, con lo que se integran de manera confusa al cuadro total del desorden. La heterogeneidad se muestra en la existencia de síntomas opuestos (pérdida/aumento de peso, insomnio/hipersomnia, agitación/retraso psicomotor) y la ausencia de jerarquía u orden de importancia en el conjunto. En tercer y último lugar, la aproximación minimiza la subjetividad. Se omite el modo concreto en el que un individuo tiene experiencia de los trastornos (Maldiney, 2001, p. 31). Así, los procedimientos de evaluación empleados 
optan por la homogeneización en cuanto a que "la sintomatología del paciente necesita ajustarse a criterios de diagnóstico preexistentes, por lo que pasan por alto las diferencias sutiles de experiencia y su significado para el paciente" (Stanghellini $e t$ al., 2019, p. 4).

Ahora bien, si el enfoque psiquiátrico es una perspectiva cuestionable por los motivos antes indicados, uno de los interrogantes que naturalmente se presenta es el siguiente: $i$ acaso es posible abordar la enfermedad mental desde un punto de vista no exclusivamente nosológico? La respuesta ante esta inquietud se localiza en la perspectiva instaurada por la psiquiatría fenomenológica, que busca entender cómo las características estructurales de la experiencia son alteradas en presencia del desorden. Frente a la limitación de la aproximación nosológica enunciada en los puntos (i) a (iii), este enfoque da cuenta de las estructuras y principios que, sin necesariamente mostrarse, subyacen a las manifestaciones particulares. Además, cuestiona la insuficiencia del saber psiquiátrico para demostrar la existencia de una relación de necesidad entre los síntomas que la disciplina vincula a un determinado trastorno (Doerr-Zegers et al., 2017).

El quehacer fenomenológico se distingue de las ciencias que analizan "hechos" contingentes (síntomas), pues estudia los elementos que caracterizan los modos de ser comprometidos en la enfermedad. Siguiendo a Heidegger (2015, p. 216), filósofos como Binswanger (1971, p. 15) describen cómo el padecimiento modifica nuestro trato habitual con el mundo. El autor establece que el saber psiquiátrico carece de un fundamento sólido, ya que conceptualiza los trastornos única y exclusivamente mediante sus síntomas, y omite que dicha manifestación concreta revela al mismo tiempo la forma de ser del sujeto que la padece (1971, p. 10). En L'Introduction à l'analyse existentielle podemos leer lo siguiente:

Desde un punto de vista fenomenológico, lo esencial de tales fenómenos psicopatológicos reside en el hecho de que nunca se ve un fenómeno aislado, sino que siempre se desarrolla en el trasfondo de un Yo, de una persona o, dicho de otro modo, siempre lo vemos como una expresión o manifestación que proviene de tal o cual persona. En el fenómeno particular, la persona se da a conocer e, inversamente, es el fenómeno (particular) ${ }^{3}$ el que hace que se penetre en la persona (1971, p. 105).
De acuerdo con esto, una indagación fenomenológica acerca de la psicopatología no devela cierta "deficiencia" de un sujeto respecto de una norma dada, sino más bien otra posible manera mediante la que este puede desenvolverse prácticamente en su entorno (Maldiney, 2007, p. 8.; Binswanger, 1971, p. 39). Si la psiquiatría convencional analiza la enfermedad mental bajo el supuesto de una idea preconcebida del ser humano que permite distinguir entre el individuo "normal" y el "enfermo" (Binswanger, 1971, p. 16), la psiquiatría fenomenológica omite toda preconcepción estática. Con ello, pretende acceder sin "prejuicios" a la forma originaria en que la enfermedad mental "aparece" ante nosotros. Así: "lo patológico no es simplemente anormal en el sentido de carecer de norma, desviarse de las normas comunes u operar en contra de ellas; también establece su propia normalidad" (Heinämaa y Taipale, 2019, p. 293). Por esta razón, el individuo que padece una psicopatología no se distingue de quienes no la experimentan en virtud de su enfermedad, sino más bien en razón de los elementos que caracterizan su experiencia (Binswanger, 1971, p. 20).

Rovaletti afirma que "si la filosofía fenomenológica investiga las estructuras universales requeridas por el aparecer de la conciencia misma, la psico(pato)logía fenomenológica investiga las estructuras empíricas que son típicas o generales para un grupo de personas" (2016, p. 139). Así, un análisis fenomenológico de la psicopatología implica la puesta en marcha de un doble movimiento. Por un lado, se busca esclarecer cuáles son los elementos típicos que caracterizan la irrupción de una determinada enfermedad mental, esto es, "cómo" se desenvuelve en el mundo el sujeto que la padece. Por otro lado, la operación aclara en qué medida dicha experiencia implica una alteración en la forma en que aquel sujeto se relacionaba con su entorno antes de la enfermedad. Mientras la psiquiatría convencional comprende la psicopatología como la disrupción en la experiencia a causa del surgimiento de una cantidad de síntomas particulares, la psiquiatría fenomenológica entiende los trastornos como la alteración parcial o íntegra que se produce en el vínculo entre un individuo y el mundo. Este enfoque, en lugar de estudiar síntomas heterogéneos sin su integración en un conjunto, logra una aproximación desde una perspectiva sistemática al establecer que lo alterado por el trastorno son las estructuras de la experiencia pensada como un todo. 
Planteado lo anterior, su gran innovación reside en la descripción de esas estructuras que subyacen a la sintomatología analizada por la psiquiatría convencional, y por medio de ello se logra mayor precisión y alcance en el abordaje de su objeto de estudio. De ello se extrae que la comprensión fenomenológica de la enfermedad apunta a un tipo de experiencia en la que el modo habitual de vincularlos con el mundo resulta modificado. En otras palabras, algunas de las actividades cotidianas se tornan imposibles con el advenimiento de este tipo de padecimiento. Havi Carel describe el cambio en los siguientes términos:

La facilidad con la cual realizamos tareas habituales a menudo desaparece en la enfermedad, en la que aparecen conductas novatas como el resultado de la pérdida de capacidades (...) y la necesidad de encontrar nuevas formas para realizar las tareas rutinarias (2016, p. 49).

Un elemento central que figura en la vivencia de trastornos como la depresión corresponde a los desórdenes de la corporización -en particular, la disrupción en el sentido de agencia-. Como veremos más adelante, uno de sus aspectos característicos para la psiquiatría fenomenológica es la alteración que implica en el vínculo corporal con nuestro entorno, lo que se traduce en una disminución en la cantidad de actividades que efectivamente podemos realizar. En el siguiente apartado ahondaremos en dicha discusión.

\section{DESORDEN DE LA CORPORIZACIÓN: EL SENTIDO DE AGENCIA}

La noción de corporización se propone apresar la compleja interconexión entre el cuerpo biológico -principalmente, la experiencia sensoriomotora del organismo-y el ambiente en la cognición. Su estudio se funda, en gran medida, en la convergencia entre las ciencias cognitivas y la fenomenología (Fuchs y Schlimme, 2009).

El enfoque de la cognición corporizada ${ }^{4}$, considerado como una aproximación no clásica ${ }^{5}$ en el área, se caracteriza por el énfasis en la estructura, postura y capacidades del cuerpo biológico del sujeto (incluyendo el cerebro) en los procesos cognitivos. De igual modo, conceptualiza el entorno como un regulador tanto directo como indirecto. En este marco, algunas aristas de estudio (por ej. Gallagher, 2000, 2007; Gallagher y Zahavi 2008) incluyen la experiencia consciente en primera persona como factor fundamental de la corporización y afirman que es estructurada por la configuración corporal. Dicha conceptualización se extiende al estudio de los trastornos mentales: gran parte de las psicopatologías son entendidas como "desórdenes de la corporización", porque se producen o expresan en el cuerpo (comprendido de manera amplia). Su irrupción significaría la existencia de una experiencia subjetiva alterada, de la mano de una disfunción neurobiológica y un menoscabo en las interacciones sociales, de manera independiente al componente originalmente afectado.

Un tipo de desorden de la corporización corresponde a la afección del sentido prerreflexivo corporizado del sí mismo y, de manera más específica, del sentido de agencia. De acuerdo con Gallagher (2000), se entiende como "la sensación de que yo soy quien está causando o generando una acción" (p. 15), es decir, la experiencia de control en el inicio o generación de una acción propia ${ }^{6}$. Este sentido involucra usualmente una conciencia fenomenológicamente atenuada (thin) (Haggard y Eitam, 2015, p. xii): las actividades intencionales cotidianas son llevadas a cabo sin una experiencia compleja o reflexiva de agencia sobre ellas. En su lugar, usualmente basta con una consciencia mínima de control intencional -aunque puede también encontrarse a nivel de conciencia explícita-.

Al menos dos distinciones son relevantes para su dilucidación. En primer lugar, se han planteado distintos niveles del sentido de agencia. Synofzik, Vosgerau y Newen (2008) diferencian entre una sensación de agencia (feeling of agency) $\mathrm{y}$ un juicio de agencia (judgement of agency). El primero refiere a una experiencia de nivel inferior que figura cuando el sujeto no se encuentra pensando explícitamente en sus acciones voluntarias. Se vincula con procesos prerreflexivos y sensoriomotores. El segundo corresponde a un juicio de nivel superior que se manifiesta al realizar atribuciones explícitas de agencia a uno mismo o a otros. Se ha relacionado con procesos cognitivos reflexivos que incluyen creencias y conocimiento contextual. El sentido prerreflexivo de ser el agente de una acción se asocia con la sensación de agencia, más básica que el juicio de agencia.

La segunda distinción importante diferencia entre el sentido de agencia y el sentido de propiedad, que se define como "la sensación de que soy yo quien experimenta una experiencia” (Gallagher, 
2000, p. 15). En otras palabras, apunta a la experiencia prerreflexiva del individuo de que es su propio cuerpo el que se está moviendo, ya sea voluntaria o involuntariamente. La distinción entre ambas, por tanto, se resume en la distinción entre ser el "sujeto" del movimiento y ser su "autor". En la experiencia de acción voluntaria los dos sentidos no son distinguibles. En efecto, al tomar intencionalmente un objeto con la mano, el individuo siente que es quien ocasiona la acción, y que su cuerpo se mueve para ejecutarla. Sin embargo, en la acción involuntaria es posible trazar la divergencia: se posee un sentido de que es uno mismo quien se mueve, pero no quien ocasiona o controla el movimiento. Por ejemplo, si el sujeto es empujado por la espalda, experimenta el movimiento como ocurrido a su propio cuerpo (por lo que presenta sentido de propiedad), pero sin sentido de agencia, porque no es la causa del movimiento ${ }^{7}$.

Si bien esta distinción puede localizarse en la consciencia reflexiva o introspectiva de nivel superior, se encuentra originariamente a nivel de experiencia fenoménica de primer orden. Por esta razón, ambos sentidos son considerados dos aspectos de la autoconsciencia prerreflexiva, que forman parte de un sentido mínimo de sí (minimal $s e l f$ ). La autoconsciencia prerreflexiva es entendida como una conciencia implícita, de primer orden y anterior a cualquier reflexión respecto de la experiencia propia. El sentido mínimo de sí, por su parte, es la conciencia básica e inmediata que el sujeto tiene de sí, sin la inclusión de una extensión temporal (Gallagher, 2000, p. 15). En otras palabras, no incluye sus vivencias o pensamientos pasados. En el enfoque de la cognición corporizada, este sí mismo es operacionalizado "como una entidad física caracterizada por el procesamiento de señales multisensoriales y motoras que generan las experiencias de propiedad sobre el propio cuerpo y agencia sobre las propias acciones" (Tsakiris, 2015 , p. 235). En particular, se ha sostenido que tanto el sentido de agencia como el de propiedad se generan primariamente en procesos sensoriales y motores, cuya distinción constituye una abstracción de la experiencia corporizada íntegra. El sentido de propiedad está vinculado con señales sensoriales aferentes -como información propioceptiva o visual-, mientras que el sentido de agencia se relaciona con señales motoras eferentes (Gallagher, 2007, p. 350; Tsakiris, 2015, p. 236). No obstante, como fenómeno cognitivo complejo, no se limita a los procesos de movimiento corporal. Otra de las fuentes a considerar es el aspecto intencional ligado al monitoreo perceptual del resultado que tuvo la acción realizada en el entorno (Gallagher, 2007; David, Newen y Vogeley, 2008).

Debido a que el origen de la distinción se halla en las aproximaciones fenomenológicas de la acción corporizada, el trabajo de Husserl es central en esta perspectiva. En primer orden, un antecedente importante de la distinción entre sentido de agencia y sentido de propiedad se encuentra en la descripción del cuerpo presentada en Ideas II. En dicha obra, el autor describe la corporalidad mediante la noción de "cuerpo propio", es decir, como aquel objeto de nuestra experiencia del que podemos disponer inmediatamente (órgano de voluntad) y que, por tanto, está sujeto a nuestra libre espontaneidad. De este modo, el cuerpo posibilita que seamos autores de diferentes acciones (sentido de agencia) en el contexto del trato práctico con el mundo (Ratcliffe, 2013, p. 579; Venebra, 2018, pp. 119-120). A modo de ilustración, considérense acciones como escribir o dibujar, que únicamente son realizables en virtud del movimiento voluntario de nuestra mano. Por esta razón, para Husserl, solo en cuanto individuos situados corporalmente en el mundo desarrollamos acciones que responden al "yo puedo" de nuestra conciencia. Así, "el sujeto que se constituye como contramiembro de la naturaleza material (...) tiene la 'capacidad' ('yo puedo') para mover libremente este cuerpo o los órganos en que se articula, y para percibir mediante ellos un mundo externo" (2014, p. 192). El filósofo añade a lo anterior que la corporalidad posee un doble carácter. Por un lado, es un cuerpo objeto (Körper) que ocupa un espacio determinado en el mundo. Por el otro, es un cuerpo vivo (Leib) que, además de obedecer al "yo puedo" de la conciencia (cuerpo propio), experimenta y siente como "suyas" -esto es, en primera persona- sus experiencias (sentido de propiedad) (Venebra, 2018, p. 112). De este modo, si el sentido de agencia y de propiedad son indistinguibles en las acciones voluntarias realizadas en la relación cotidiana con nuestro entorno, con la irrupción de algunos trastornos mentales resultan profundamente alterados (Husserl, 2014, p. 192). Lo anterior establece las bases para pensar y describir ciertos tipos de experiencias que son evidenciadas como "nuestras" (sentido de propiedad) mas no como el resultado de nuestra libre espontaneidad (sentido de agencia). 
En segundo orden, la descripción del sentido de agencia como conciencia "prerreflexiva" encuentra un antecedente importante en la distinción husserliana entre "conciencia objetivante" y "conciencia preobjetivante" presentada -entre otras obras-en el marco de sus Lecciones de fenomenología de la conciencia interna del tiempo. El autor establece que a toda experiencia en la que nos relacionamos conscientemente con un determinado objeto subyace cierto saber prerreflexivo, mediante el que nos descubrimos como los sujetos que se vinculan con dicho objeto ${ }^{8}$. Volviendo al ejemplo de la acción de escribir o dibujar, podemos afirmar que el hecho de relacionarnos prácticamente con los objetos que nos permiten desarrollar el acto -como, por ejemplo, un lápiz- supone un tipo de consciencia preobjetivante en la que lo mentado somos nosotros mismos en cuanto sujetos que realizan aquella acción. En otros términos, el acto reflexivo de tomar un lápiz involucra un acto prerreflexivo por medio del que nos sabemos como los agentes del movimiento (Gallagher y Zahavi, 2008, p. 159).

El sentido de agencia puede contribuir al conocimiento del repertorio conductual, y resulta relevante en conceptos como "elección libre" y "responsabilidad". Además, la retroalimentación intencional ocurrida al observar la influencia de las acciones propias en el ambiente permite esperar recompensas o castigos ante la producción de un resultado determinado, o conocer las posibilidades que ofrece un entorno específico (Haggard y Eitam, 2015, p. xii). Asimismo, constituye una herramienta teórica útil para entender los perfiles de algunas psicopatologías en las que se desarrollan alteraciones en la estructura de la autoconsciencia básica, como en el caso de la depresión. Este punto quedará clarificado en el próximo apartado, donde analizaremos algunas de sus posibles disrupciones fenomenológicas.

\section{ALTERACIONESFENOMENOLÓGICAS DEL SENTIDO DE AGENCIA EN LA DEPRESIÓN}

Los sujetos con depresión sufren diversas alteraciones fenomenológicas en el modo en que se vinculan con el mundo. La incapacidad de encontrar alegría o placer en cualquier situación, la desconexión emocional respecto de los congéneres o la pérdida de metas vitales son solo algunos ejemplos que caracterizan su presencia. En particular, las alteraciones en el sentido de agencia se posicionan como factor central ${ }^{9}$.
En primer lugar, gran parte de los testimonios indican la prevalencia de un sentimiento de pérdida del sentido de control -también conocido como "control percibido"-. Este es un atributo cognitivo definido como "la creencia que el individuo tiene acerca de la naturaleza del control sobre factores y eventos situacionales" (Weems y Silverman, 2006, p. 117), y ha sido sistemáticamente vinculado con la depresión en la literatura especializada. En los individuos afectados, la habilidad para actuar se encuentra drásticamente disminuida, lo que se extiende incluso a actividades cotidianas $-y$ fundamentales-como el aseo personal. Así, por ejemplo, un testimonio sostiene que:

\section{Cuando estoy deprimido, cada trabajo parece más grande y más difícil. Ningún contratiempo me parece algo fácil de solucionar o superar, sino un gran obstáculo. Los acontecimientos parecen más caóticos y fuera de mi control: si no logro algún objetivo, parecerá que alcanzarlo está para siempre más allá de mis capacidades (Paciente citado en Law, 2009, p. 355).}

La persona se percibe como imposibilitada para desenvolverse en el mundo. Esta imposibilidad es atribuida, en muchos casos, a un elemento externo al sujeto mismo, como puede advertirse en el testimonio siguiente:

Tengo un sentimiento de imprevisibilidad y falta de control sobre algo que tiene vida propia que contradice mi sentimiento de dominio. Y eso lo sé ahora. He tenido esta experiencia durante tanto tiempo que voy a estar arriba y luego abajo, y supongo que eso lo hace un poco más fácil. Quiero decir, sé que va a suceder. Está fuera de mi control $y$, por lo tanto, no debería sentirme tan mal cuando pase porque es parte del ritmo de mi vida, supongo ${ }^{10}$ (Karp, 1996, pp. 124-125).

Aquí el sujeto atribuye la agencia a "algo" que tiene "vida propia". En casos en donde se presentan ideas suicidas, el sentimiento de pérdida de control en ocasiones aparece junto con una pugna por el dominio entre el individuo y sus ideas invasivas, que son reconocidas como emitidas por "otra parte del sí mismo" en el siguiente ejemplo:

Cuando me siento bien, no tengo conciencia de ninguna división de mi ser en partes. Solo opero como un ser normal y funcional. Sin 
embargo, si estoy muy deprimida o tengo ideas suicidas como efecto secundario de la medicación, a veces pienso que la única manera de resolver mis problemas es no estar más aquí. Es entonces cuando me parece sentir la dislocación. Se siente como que el pensador en mí (tal vez sea mi 'yo' normal) está bastante seguro de que el suicidio no es una solución, pero ese lado de mí es consciente de algo parecido a otra entidad dentro de mí que está emitiendo pensamientos sobre lo que considera la solución fácil: acabar con las cosas $^{11}$ (Paciente citado en Benson, Gibson y Brand, 2014, p. 44).

En segundo lugar, y en directa relación con lo anterior, los sujetos con depresión poseen a menudo un sentimiento de pasividad patológica. Ello se vincula con la presencia de indefensión aprendida (learned helplessness) (Abramson, Seligman y Teasdale, 1978), esto es, la manifestación de un comportamiento pasivo producto de la experiencia previa de eventos incontrolables. La pasividad e indefensión se observan en el siguiente testimonio: "Alguien me preguntó una vez cómo se sentía. Perdí el equilibrio, dije. Se sentía como si hubiera perdido el equilibrio. Me caí de bruces y no pude volver a levantarme" (Brampton, 2008, p. 42).

Para las personas con depresión, usualmente el mundo no es experimentado como un locus de diversas posibilidades. En su lugar, se sienten impotentes e incapaces ante él, y comúnmente lo reflejan en frases como "perder el balance", "caer en un agujero negro" o "ahogarse". Además, puede presentarse como atemorizante u opresor. Por ejemplo:

Los objetos ordinarios -sillas, mesas y similares- poseían una cualidad aterradora y amenazante que es muy difícil de describir vívidamente en la forma en que entonces me afectaba. Era como si viviera en una especie de infierno, que no contenía nada de lo que pudiera obtener alivio o consuelo (Paciente citado en Rowe, 1978, pp. 269-270).

La pasividad patológica y la pérdida de control frente al entorno desembocan en un individuo temeroso, abrumado o sofocado. Estos sentimientos, como se observa en el reporte, no siempre se presentan ante una situación concreta, sino que afectan la manera misma de experimentar el ambiente. Como resultado, incluso los objetos cotidianos sofocan o atemorizan.

En tercer lugar, al considerar la descripción husserliana del cuerpo propio como "órgano de voluntad" desarrollada en Ideas II se extrae que otro de los aspectos fundamentales de la depresión es la chrematización. Esta refiere a un sentimiento de desvitalización por medio del que un individuo evidencia una disminución en su capacidad de agencia. Si nuestro vínculo corporal con el mundo va acompañado habitualmente de un sentimiento de espontaneidad, con la irrupción de la depresión el cuerpo deviene una realidad agónica para la que incluso actividades básicas como el levantarse de la cama para trabajar resultan una meta imposible de conseguir (Doerr-Zegers et al., 2017, p. 277). Por ello, una persona con depresión, en lugar de experimentar el control de su cuerpo, lo percibe como un objeto (chrema) del que es incapaz de disponer. En la siguiente cita figura una explicación de esta vivencia:

El primer fenómeno fundamental que constituye una disrupción de la corporización es la alteración de la relación del sujeto con su propio cuerpo. Se trata de una disrupción de la sensibilidad (el modo de encontrarse o sentirse en el cuerpo propio), Die Befindlichkeit en alemán, como es usado en la psicopatología clásica y la medicina antropológica (...). Desde la perspectiva del paciente, el cambio en la experiencia de su propio cuerpo se muestra de múltiples formas, entre las cuales el estado de ánimo deprimido (primer criterio del DSM) y la pérdida de energía (sexto criterio del $D S M$ ) representan solo una parte del complejo fenómeno. Los pacientes manifiestan muchos de estos síntomas, que están interconectados porque todos son manifestaciones de la alteración de la Befindlichkeit: ansiedad, pesadez de las extremidades, dolor en varias partes del cuerpo, opresión precordial, el clásico globus melancholicus, sensación de frío o náuseas, etc. (Doerr-Zegers et al., 2017 , p. 277).

De esta manera la chrematización, en cuanto disminución del sentimiento de disposición de nuestro cuerpo, representa un elemento primordial en las alteraciones de la experiencia que subyacen a la depresión. Si queremos describir "afirmativamente" la pérdida, podemos establecer que mienta un peculiar tipo de cansancio en el que lo que pesa y fatiga no es 
la realización de una acción determinada, sino más bien la dificultad de ser agentes en el contexto de la relación con el mundo. Por esta razón, el cansancio de la persona con depresión debe ser diferenciado de aquel que, por ejemplo, experimenta un deportista luego de ejecutar su rutina de ejercicios. En L'existence en question dans la dépression et la mélancolie, Henri Maldiney señala que uno de los elementos que caracteriza toda experiencia depresiva es la "lasitud" o "pesantez" mediante la que aparece nuestra propia corporalidad. Si cotidianamente el cuerpo se presenta como el órgano que nos permite realizar diferentes acciones, con el surgimiento de la depresión se revela como uno que "pesa" al punto de no poder disponer de él (Maldiney, 2007, p. 82). El testimonio presentado por Outi Benson en su conferencia Using the Grounded Theory Method to explore Emotional Experience associated with Self-cutting da cuenta de la chrematización en los siguientes términos:

Me acuesto en la cama por años temiendo meterme en la ducha y luego, cuando finalmente estoy en la ducha, termino estando allí por años temiendo salir (...). La sensación de temor no es un miedo o temor a que algo específico suceda, es más bien como sentirse discapacitado de alguna forma, como que el esfuerzo y la idea de pasar a la siguiente cosa, sea cual sea, se siente demasiado abrumador. (...) Es casi como si estuviera allí pero no pudiera tocar nada o conectarme. Todo requiere demasiado esfuerzo y no soy realmente capaz. de hacer nada. Si noto que algo necesita ser limpiado o movido, es como si estuviera fuera de alcance (...) como si pudiera ver muchos detalles, pero no ser parte de ello. Supongo que sentirse desconectado es la mejor manera de describirlo ${ }^{12}$ (Paciente citado en Benson, 2010, recuperado por Ratcliffe, 2013, p. 585).

De acuerdo con esta experiencia, una corporalidad chrematizada producto de la depresión es una que deviene la mera espectadora de un entorno del que es incapaz de formar parte.

Debido a que toda chrematización es una disrupción en la autoconsciencia corporal, va acompañada ineludiblemente por un cuarto criterio: el fenómeno de la inhibición, entendido como la pérdida de la espontaneidad por la que nos vinculamos habitualmente con el mundo (Doerr-Zegers et al., 2017, p. 277). El surgimiento del trastorno altera la capacidad de realizar diferentes actos cuyo soporte es nuestro cuerpo. Como consecuencia de lo anterior, aquel "yo puedo" con el que Husserl describe la relación con el mundo da paso a un sentimiento de incapacidad (chrematización) que se traduce en una "inhibición" o disminución concreta de la agencia, es decir, del conjunto de actividades que efectivamente podemos realizar (Ratcliffe, 2013, p. 586).

Esbozada la definición general de la inhibición, es importante realizar ciertas precisiones respecto de la diferencia que tiene con la chrematización. Si la chrematización refiere a un sentimiento general de incapacidad, la inhibición apunta al modo concreto en que se expresa dicha incapacidad. Se trata del saber mediante el que nos damos cuenta de que las actividades que podíamos realizar con relativa facilidad se convierten en tareas imposibles de ejecutar. Matthew Ratcliffe establece que, en la inhibición, "hay una especie de distanciamiento en el que el mundo sigue ofreciendo posibilidades para los otros, que se presentan como 'imposibles para mí"' (2013, p. 579). De acuerdo con esto, la alteración en el sentido de agencia tiene como consecuencia inevitable una transformación en el modo en que nos aparece el entorno. Recogiendo diversos reportes, Dorothy Rowe observa que, ante esta situación, "cada persona describe la experiencia como estar encerrado" (1978, p. 30). El siguiente testimonio da cuenta de ello: "Quiero llegar al mundo, pero no está ahí para llegar a él ... Nada puede entrar o salir de mi psique. Estoy emocionalmente aislado, en una isla con mar alrededor y sin posibilidad de rescate" (Paciente citado en Benson, Gibson y Brand, 2014, p. 46).

Centrando la atención en el "estar encerrado" involucrado en la inhibición, el sentimiento de chrematización y su consecuente inhibición provocan que el mundo no aparezca como un "horizonte de posibilidades" en el que desarrollamos diferentes actos (Ratcliffe, 2013, p. 579). Lo anterior puede ser entendido en la medida que, en el trastorno depresivo, predomina un sentimiento de incapacidad por el que la comprensión misma de cualquier "posibilidad de acción" resulta algo inaccesible para las personas que lo padecen ${ }^{13}$.

A modo de sumario, existen al menos cuatro rasgos que dan cuenta de la disrupción del sentido de agencia presente en sujetos con trastorno depresivo mayor, a saber: (i) sentimiento de pérdida de control, (ii) sentimiento de pasividad patológica, (iii) chrematización e (iv) inhibición. El estudio interdisciplinar del desorden de la corporización 
indica que se trata de un fenómeno estructural que caracteriza el modo de ser del sujeto con depresión.

\section{CONCLUSIÓN}

El objetivo del presente artículo se enfocó en el análisis de las alteraciones en el sentido de agencia en sujetos con depresión por medio del estudio de testimonios registrados en la literatura. Identificamos cuatro rasgos que apuntan a su disrupción: pérdida de control, pasividad patológica, chrematización e inhibición. La consideración del estudio interdisciplinar de la corporización y sus alteraciones posee implicancias en, al menos, dos niveles: un nivel conceptual relacionado con la noción de psicopatología, y un nivel metodológico acerca del abordaje integral de los trastornos mentales.

Respecto del nivel conceptual, este tipo de análisis posibilita un cambio en la noción de desorden como un conjunto de signos y síntomas que generan sufrimiento significativo y déficit en el desempeño. Por un lado, el enfoque de la cognición corporizada, por medio del énfasis en el rol de los procesos y actividades corporales en la cognición, contribuye a explicar la extensión de los síntomas psicopatológicos al cuerpo. Por otro, la fenomenología permite estudiar los aspectos de la experiencia consciente en primera persona que apuntan a la naturaleza corporizada de la cognición, y los cambios que surgen a raíz de la enfermedad. La noción de trastorno mental resultante incluye las disrupciones en esta experiencia en primera persona $\mathrm{y}$, en particular, en su capacidad de poder vivenciar y comportarse (Blankenburg, 1983) sintiéndose agente de sus acciones.

En relación con el nivel metodológico, el estudio interdisciplinario de la corporización presenta una crítica al enfoque nosológico que no apunta a su sustitución. Frente a la reducción de la psicopatología a la manifestación de síntomas sin relación de necesidad entre sí, el análisis da cuenta de los elementos transversales en los que se funda la sintomatología, con lo que aborda las estructuras de la experiencia alteradas en dicho tipo de vivencias. Sin embargo, las objeciones respecto de la insuficiencia de manuales diagnósticos como el $D S M-V$ no sugieren el reemplazo de un enfoque por otro. A causa de que el manual es nosológico y no fenomenológico, su objetivo radica en facilitar el diagnóstico para elaborar un plan de tratamiento. Por tanto, los síntomas incluidos no pretenden capturar la complejidad de los trastornos en definiciones integrales. En lugar de una sustitución, el hincapié realizado en la importancia de aspectos como el sentido de agencia instan a mantener la interdisciplinariedad mediante la complementación entre aproximaciones. Mediante la exégesis de las estructuras comprometidas con el surgimiento de estas manifestaciones y el modo concreto en que afectan al individuo, se posibilita una mayor consistencia para la sintomatología. Siguiendo esta perspectiva, es posible acercarse progresivamente a la definición integral de la que carece el $D S M-V$.

$\mathrm{El}$ análisis de las alteraciones en el sentido de agencia en sujetos con depresión realizado en el presente artículo puede ser considerado como una primera aproximación a dicha definición integral. Por ello, sus limitaciones se encuentran principalmente vinculadas a la restricción en el número de rasgos relacionados con las disrupciones en el sentido de agencia que fueron abordados y en el número de testimonios asociados. Algunas de las futuras directrices de investigación que sugerimos son las siguientes:

1. Identificación de otros rasgos que apunten a la disrupción del sentido de agencia en personas con depresión, así como de las relaciones que existen entre ellos. Si bien se inscriben en una experiencia unitaria, su distinción fenomenológica permite una mayor comprensión del fenómeno, así como una mejor acomodación de los testimonios.

2. Examen de otras dimensiones de la depresión desde la perspectiva interdisciplinar de la corporización. Algunos ejemplos corresponden al estudio del aspecto temporal y el aspecto social que caracterizan las vivencias expresadas en los testimonios.

3. Análisis de las similitudes y diferencias entre los rasgos característicos de la disrupción del sentido de agencia en la depresión y en otros perfiles como la esquizofrenia o el trastorno obsesivo compulsivo.

En suma, la consideración de los elementos expuestos puede constituir un aporte teórico, conceptual y metodológico tanto para los profesionales de la salud mental (psiquiatras y psicólogos) como para los encargados de elaborar políticas públicas respecto de este tópico. 


\section{REFERENCIAS}

Abramson, L.Y., Seligman, M.E. y Teasdale, J.D. (1978). Learned Helplessness in Humans: Critique and Reformulation. Journal of Abnormal Psychology 87 (1), 49-74.

American Psychiatric Association (2013). Diagnostic and Statistical Manual of Mental Disorders ( $5^{\mathrm{a}}$ ed.). American Psychiatric Publishing.

Benson, O. (2010). Using the Grounded Theory Method to Explore Emotional Experience Associated with Self-cutting. Conferencia Julio 2010. Citado en Ratcliffe, M. (2013). Depression and the Phenomenology of Free Will. The Oxford Handbook of Philosophy and Psychiatry. Oxford University Press, 574-591.

Benson, O., Gibson, S. y Brand, S.L. (2014). The Experience of Agency in the Feeling of Being Suicidal. En M. Ratcliffe y A. Stephan (Eds.), Depression, Emotion and the Self. Philosophical and Interdisciplinary Perspectives. Imprint Academic, 37-58.

Binswanger, L. (1971). De la Phénoménologie. Introduction à l'Analyse Existentielle, Minuit, 79-117.

Blankenburg, W. (1983). La psicopatología como ciencia básica de la psiquiatría [Psychopathology as a basic science of psychiatry]. Revista Chilena de Neuro-psiquiatría 21 (3), 177-188.

Brampton, S. (2008). Shoot the Damn Dog: A Memoir of Depression. Bloomsbury.

Carel, H. (2016). Phenomenology of Illness. Oxford University Press.

Centro de Estudios de Conflicto y Cohesión Social - COES (2018). Resultados Primera Ola, Estudio Longitudinal Social de Chile (ELSOC). Módulo 6: Salud y bienestar. Salud Mental en el Chile de hoy. Notas COES de Política Pública No 15 . ISSN: 0719-8795. Santiago, Chile: COES. Recuperado de: http://www. elsoc.cl/publicaciones-elsoc/informes

David, N., Newen, A. y Vogeley, K. (2008). The 'Sense of Agency' and its Underlying Cognitive and Neural Mechanisms. Consciousness and Cognition 17 (2), 523-534.

Doerr-Zegers, O., Irarrázaval, L., Mundt, A. y Palette, V. (2017). Disturbances of Embodiment as Core Phenomena of Depression in Clinical Practice. Psychopathology 50 (4), 273-281.

Fuchs, T. y Schlimme, J.E. (2009). Embodiment and Psychopathology: A Phenomenological Perspective. Current Opinion in Psychiatry 22 (6), 570-575.

Gallagher, S. (2000). Philosophical Conceptions of the Self: Implications for Cognitive Science. Trends in Cognitive Sciences 4 (1), 14-21.

Gallagher, S. (2007). The Natural Philosophy of Agency. Philosophy Compass 2 (2), 347-357.

Gallagher, S. y Zahavi, D. (2008). The Phenomenological Mind. An Introduction to Philosophy of Mind and Cognitive Science. Routledge.

Haggard, P. y Eitam, B. (2015). Introduction. En P. Haggard y B. Eitam (Eds.), The Sense of Agency. Oxford University Press, xi-xvi.

Heidegger, M. (2015). Ser y Tiempo. Editorial Universitaria.
Heinämaa, S. y Taipale, J. (2019). Normality. En G. Stanghellini, M.R. Broome, A.V. Fernandez, P. Fusar-Poli, A. Raballo y R. Rosfort (Eds.), The Oxford Handbook of Phenomenological Psychopathology. Oxford University Press, 284-296.

Husserl, E. (2002). Lecciones de fenomenología de la conciencia interna del tiempo. Trotta.

Husserl, E. (2014). Ideas relativas a una fenomenología pura y una filosofía fenomenológica. Libro segundo: Investigaciones fenomenológicas sobre la constitución. Fondo de Cultura Económica.

Karp, D.A. (1996). Speaking of Sadness: Depression, Disconnection, and the Meanings of Illness. Oxford University Press.

Law, I. (2009). Motivation, Depression and Character. En M.R. Broome y L. Bortolotti (Eds.), Psychiatry as Cognitive Neuroscience: Philosophical Perspectives. Oxford University Press, 351-364.

Maldiney, H. (2001). L'homme dans la psychiatrie. Revue de psychothérapie psychanalytique de groupe 36, 31-46.

Maldiney, H. (2007). Penser l'homme et la folie. Millon.

Moore, J.W. (2016). What is the Sense of Agency and Why Does it Matter? Frontiers in Psychology, 7, 1272.

OCDE (2014), Suicides. En OECD Factbook 2014: Economic, Environmental and Social Statistics. OECD Publishing, https:// doi.org/10.1787/factbook-2014-99-en

Ratcliffe, M. (2013). Depression and the Phenomenology of Free Will. The Oxford Handbook of Philosophy and Psychiatry. Oxford University Press, 574-591.

Rovaletti, M.L. (2016). La significación de la fenomenología en psiquiatría. Revista de Filosofía, 57, 131-142.

Rowe, D. (1978). The Experience of Depression. John Wiley and Sons.

Stanghellini, G., Broome, M.R., Fernandez, A.V., Fusar-Poli, P., Raballo, A. y Rosfort, R. (2019). Introduction. En G. Stanghellini, M.R. Broome, A.V. Fernandez, P. Fusar-Poli, A. Raballo y R. Rosfort (Eds.), The Oxford Handbook of Phenomenological Psychopathology. Oxford University Press, 1-8.

Synofzik, M., Vosgerau, G. y Newen, A. (2008). Beyond the Comparator Model: A Multifactorial Two-Step Account of Agency. Consciousness and Cognition 17 (1), 219-239.

Tsakiris, M. (2015). The Relationship Between Agency and Body Ownership. Additive or Independent? En P. Haggard y B. Eitam (Eds.), The Sense of Agency. Oxford University Press, 235-249.

Venebra, M. (2018). Husserl. Cuerpo propio y alienación. Investigaciones Fenomenológicas, 15, 109-132.

Weems, C.F. y Silverman, W.K. (2006). An Integrative Model of Control: Implications for Understanding Emotion Regulation and Dysregulation in Childhood Anxiety. Journal of Affective Disorders 91 (2-3), 113-124.

Zúñiga, B. (en prensa). La manifestación hylética de la intencionalidad objetivante. Un motivo husserliano en la filosofía de Michel Henry. Revista Psicologia USP. Michel Henry e clínica, 2. 


\section{NOTAS}

1 Todas las traducciones presentadas en este artículo son de nuestra autoría.

2 Otras críticas a manuales de diagnóstico como el DSM pueden ser encontradas en Doerr-Zegers et al. (2017) y Stanghellini et al. (2019).

3 El paréntesis es nuestro.

4 La cognición corporizada no corresponde a un enfoque único, sino que engloba una variedad de aproximaciones. Estas presentan discordancias en el nivel de corporización considerado y en las críticas al enfoque clásico que sostienen, entre otros puntos teóricos. Para efectos del presente artículo definiremos la vertiente únicamente mediante su tesis central, a saber, que diversos aspectos de la cognición dependen de las características del cuerpo físico del agente (más allá del cerebro). Por ejemplo, se ha sostenido que la postura erguida de los humanos determina en gran medida sus capacidades perceptuales y motoras.

5 La cognición corporizada problematiza el clásico Modelo Computacional-Representacional de la Mente. Según este, la cognición es computacional y representacional. Por un lado, las representaciones mentales corresponderían a símbolos que pueden ser evaluados semánticamente -es decir, su contenido es susceptible de atribución de valor de verdad- de acuerdo con su relación con el ambiente y con otros símbolos. Estos son similares a las estructuras de datos de los lenguajes de programación -cadenas de letras y números, listas y árboles-. Por otro lado, los procesos computacionales serían algoritmos: procesos mecánicos que trabajan sobre esas estructuras de datos. Así, la cognición es entendida como un procesamiento de información vía la manipulación de símbolos, lo que tiene como centro la llamada "metáfora del computador". El funcionalismo (postura en donde los estados mentales son determinados por su función en lugar de su constitución) contribuyó a sostener la irrelevancia de la implementación física, siempre que el programa de software y la información se ejecuten en un hardware adecuado. En este contexto, por tanto, se aboga por la posibilidad de estudiar la cognición separada del cuerpo biológico -y, por supuesto, del contexto sociocultural también-.

6 Se han planteado diversas teorías y diseños experimentales para dar cuenta del sentido de agencia. Para una revisión véase, por ejemplo, Gallagher (2007), David, Newen y Vogeley (2008) y Moore (2016).

7 En la literatura se han propuesto distintas relaciones entre el sentido de agencia y sentido de propiedad. Para una revisión de los experimentos psicológicos y de neuroimagen llevados a cabo, así como sus modelos resultantes, véase Tsakiris (2015).

8 En el contexto de esta obra, y en sintonía con lo señalado en el parágrafo 35 de Ideas I, Husserl establece el carácter objetivante de la conciencia intencional, esto es, el hecho de que toda conciencia es una conciencia reflexiva que apunta hacia un objeto determinado. Sin embargo, y en el marco de la resolución del problema relativo a la dimensión temporal de la conciencia que orienta gran parte de los esfuerzos de las Lecciones, el camino no se encuentra exento de dificultades. Si sabemos que los fenómenos se manifiestan intencionalmente a la conciencia, la pregunta por el aparecer mismo de la intencionalidad -es decir, de los actos mediante los que mentamos reflexivamente los objetos de nuestra experiencia- resulta una aporía que la fenomenología husserliana no puede resolver en principio. Si todo aparecer implica una conciencia intencional de carácter reflexivo, la manifestación misma de la intencionalidad nos remitiría bajo esta lógica a otra conciencia reflexiva que la miente, la que a su vez requeriría de otra conciencia que la piense y así sucesivamente. De este modo, al disponer únicamente de un saber reflexivo, el aparecer de nuestros actos intencionales nos conduciría hacia un regreso al infinito (Zúñiga, en prensa). Teniendo a la vista tal dificultad el autor indica que, si bien la conciencia intencional apunta reflexivamente a diversos objetos, a dicha manifestación subyace un saber prerreflexivo mediante el que tenemos conciencia de nuestros distintos actos intencionales. Así, si lo que aparece frente a la conciencia intencional es un objeto, el aparecer mismo de la conciencia no es "nada objetivo en el tiempo" (Husserl, 2002, p. 95). En suma, toda conciencia reflexiva supone una conciencia prerreflexiva.

9 Es admisible notar que la pérdida o disrupción patológica del sentido de agencia varía en dependencia de cuál es el componente dañado por la psicopatología presentada por el individuo (Gallagher, 2007, p. 355), por lo que es una fuente de debate en la literatura especializada. En este artículo, no obstante, nos centraremos en las alteraciones fenomenológicas que se presentan.

10 El uso de cursiva es nuestro.

11 El uso de cursiva es nuestro.

12 El uso de cursiva es nuestro.

13 Respecto de este punto, nos parece importante mencionar que, en la aproximación fenomenológica a la depresión desarrollada por Henri Maldiney en L'existence dans la dépression et dans la mélancolie, el autor establece que uno de los caracteres comunes a cualquier trastorno depresivo reside en la alteración temporal que implica. Más precisamente, la experiencia temporal de una persona con depresión puede ser descrita en términos de un "presente eternizado" que cierra al sujeto tanto a la dimensión del pasado como a las posibilidades que se descubren mediante la dimensión del futuro. En suma, no hay más tiempo que aquel presente en el que experimenta su incapacidad para ejecutar cualquier acción (Maldiney, 2007, pp. 77-78). 